\title{
Update of the Used Fuel Disposition Campaign Implementation Plan
}

\section{Fuel Cycle Research \& Development}

Prepared for

U.S. Department of Energy Used Fuel Disposition

Shannon M. Bragg-Sitton Idaho National Laboratory

Jens Birkholzer

Lawrence Berkeley National Laboratory

Robert MacKinnon, Kevin McMahon, Sylvia Saltzstein, Ken Sorenson, Peter Swift, Sandia National Laboratories

September 2014

FCRD-UFD-2014-000047

INL/EXT-14-31606 



\section{DISCLAIMER}

This information was prepared as an account of work sponsored by an agency of the U.S. Government. Neither the U.S. Government nor any agency thereof, nor any of their employees, makes any warranty, expressed or implied, or assumes any legal liability or responsibility for the accuracy, completeness, or usefulness, of any information, apparatus, product, or process disclosed, or represents that its use would not infringe privately owned rights. Reference herein to any specific commercial product, process, or service by trade name, trade mark, manufacturer, or otherwise, does not necessarily constitute or imply its endorsement, recommendation, or favoring by the U.S. Government or any agency thereof. The views and opinions of authors expressed herein do not necessarily state or reflect those of the U.S. Government or any agency thereof. 



\section{FCT DOCUMENT COVER SHEET ${ }^{1}$}

Name/Title of

Deliverable/Milestone/Revision No.

Work Package Title and Number

Work Package WBS Number

Responsible Work Package Manager
Update of the Used Fuel Disposition Campaign Implementation Plan; M2FT-14IN0801011

Management and Integration - INL; FT-14IN080101

$\underline{1.02 .08 .01}$

Shannon Bragg-Sitton

(Name/Signature)

Date Submitted

\begin{tabular}{|l|l|l|l|l|}
\hline $\begin{array}{l}\text { Quality Rigor Level for } \\
\text { Deliverable/Milestone }\end{array}$ & $\square$ QRL-3 & $\square$ QRL-2 & $\begin{array}{l}\square \text { QRL-1 } \\
\text { Nuclear Data }\end{array}$ & $\begin{array}{l}\square \text { Lab/Participant } \\
\text { Qrogram (no } \\
\text { additional FCT QA } \\
\text { requirements) }\end{array}$ \\
\hline
\end{tabular}

This deliverable was prepared in accordance with

Idaho National Laboratory

QA program which meets the requirements of
DOE Order 414.1
NQA-1-2000
Other

\section{This Deliverable was subjected to:}

Technical Review

Technical Review (TR)

\section{Review Documentation Provided}

Signed TR Report or,

Signed TR Concurrence Sheet or,

Signature of TR Reviewer(s) below

below

\section{Name and Signature of Reviewers}

NOTE 1: Appendix E should be filled out and submitted with the deliverable. Or, if the PICS:NE system permits, completely enter all applicable information in the PICS:NE Deliverable Form. The requirement is to ensure that all applicable information is entered either in the PICS:NE system or by using the FCT Document Cover Sheet.

NOTE 2: In some cases there may be a milestone where an item is being fabricated, maintenance is being performed on a facility, or a document is being issued through a formal document control process where it specifically calls out a formal review of the document. In these cases, documentation (e.g., inspection report, maintenance request, work planning package documentation or the documented review of the issued document through the document control process) of the completion of the activity, along with the Document Cover Sheet, is sufficient to demonstrate achieving the milestone. If QRL 1,2, or 3 is not assigned, then the Lab / Participant QA Program (no additional FCT QA requirements) box must be checked, and the work is understood to be performed and any deliverable developed in conformance with the respective National Laboratory I Participant, DOE or NNSA-approved QA Program. 
Used Fuel Disposition Campaign Implementation Plan

FCRD-UFD-2014-000047, INL-EXT-14-31606

This page is intentionally blank. 


\section{Table of Contents}

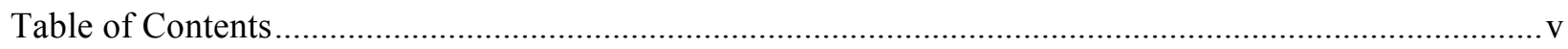

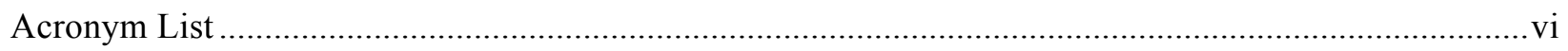

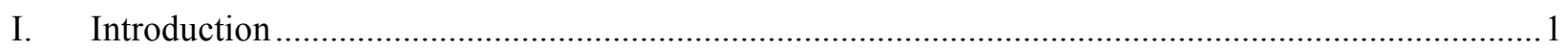

II. Fuel Cycle Technologies Program Mission and Objectives ........................................................... 1

III. DOE Strategy for the Management and Disposal of UNF and HLW …....................................... 3

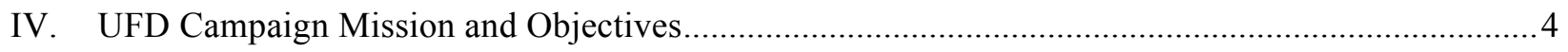

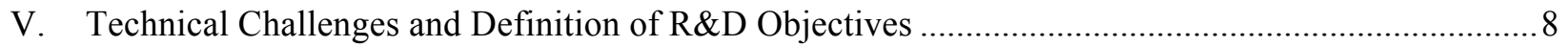

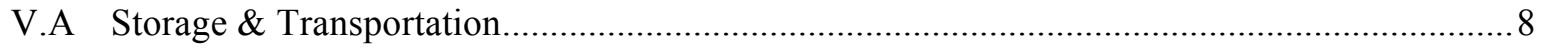

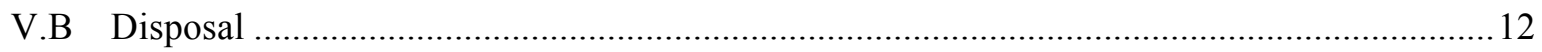

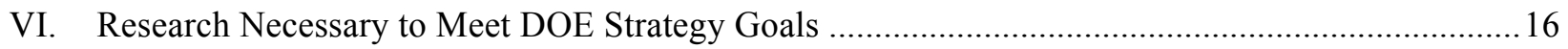

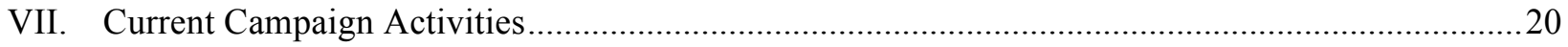

VII.A Campaign Management and Integration .......................................................................20

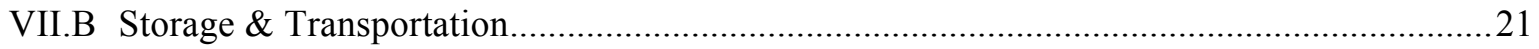

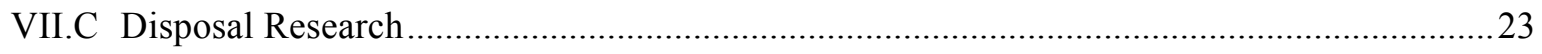

VII.D High Burnup Dry Storage Cask Demonstration ..........................................................26

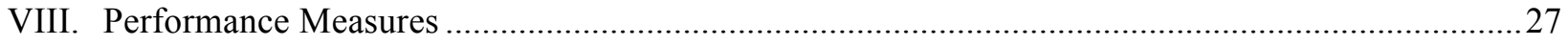

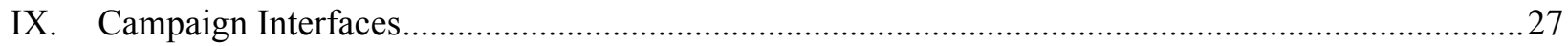




\section{Acronym List}

\begin{tabular}{|c|c|}
\hline CISF & Consolidated Interim Storage Facility \\
\hline DECOVALEX & DEvelopment of COupled models and their VALidation against EXperiments \\
\hline DNTD & Deputy National Technical Director \\
\hline DOE & Department of Energy \\
\hline DOE-NE & DOE Office of Nuclear Energy \\
\hline $\mathrm{DPC}$ & Dual-Purpose Canister \\
\hline DR & Disposal Research \\
\hline EBS & Engineered Barrier System \\
\hline EIS & Environmental Impact Statement \\
\hline EPRI & Electric Power Research Institute \\
\hline FCRD & Fuel Cycle Research and Development \\
\hline FCT & Fuel Cycle Technologies \\
\hline FEBEX & Full-scale Engineering Barrier EXperiments \\
\hline FEIS & Final Environmental Impact Statement \\
\hline FEPs & Features, Events and Processes \\
\hline GIS & Geographical Information System \\
\hline HBU & High Burnup \\
\hline HLW & High Level Waste \\
\hline ISFSI & Independent Spent Fuel Storage Installation \\
\hline KAERI & Korean Atomic Energy Research Institute \\
\hline KURT & KAERI Underground Research Tunnel \\
\hline $\mathrm{NCT}$ & Normal Conditions of Transport \\
\hline NEUP & Nuclear Energy University Program \\
\hline NFSTPP & Nuclear Fuel Storage and Transportation Program Plan \\
\hline $\mathrm{NRC}$ & Nuclear Regulatory Commission \\
\hline NTD & National Technical Director \\
\hline PA & Performance Assessment \\
\hline QA & Quality Assurance \\
\hline $\mathrm{R} \& \mathrm{D}$ & Research \& Development \\
\hline S\&T & Storage \& Transportation \\
\hline $\mathrm{SCC}$ & Stress Corrosion Cracking \\
\hline SET & Separate Effects Tests \\
\hline
\end{tabular}


Used Fuel Disposition Campaign Implementation Plan

FCRD-UFD-2014-000047, INL/EXT-14-31606

September 2014

vii

SST Small-Scale Tests

UFD Used Fuel Disposition

UFDC Used Fuel Disposition Campaign

UNF Used Nuclear Fuel

UQ Uncertainty Quantification

URL Underground Research Laboratory 



\section{Introduction}

This Update to the Used Fuel Disposition Campaign Implementation Plan provides summary level detail describing how the Used Fuel Disposition Campaign (UFDC) supports achievement of the overarching mission and objectives of the Department of Energy Office of Nuclear Energy Fuel Cycle Technologies Program, building on work completed in this area since 2009.

This implementation plan begins with the assumption of target dates that are set out in the January 2013 DOE Strategy for the Management and Disposal of Used Nuclear Fuel and High-Level Radioactive Waste (http://energy.gov/downloads/strategy-management-and-disposal-used-nuclearfuel-and-high-level-radioactive-waste). These target dates and goals are summarized in section III.

This implementation plan will be maintained as a living document and will be updated as needed in response to available funding and progress in the Used Fuel Disposition Campaign and the Fuel Cycle Technologies Program.

\section{Fuel Cycle Technologies Program Mission and Objectives}

Nuclear energy is a prominent part of a reliable, sustainable energy mix and has demonstrated remarkable performance over decades of use. Despite this key role, nuclear energy use presents challenges including mounting stockpiles of used nuclear fuel (UNF) and high-level radioactive waste (HLW) and a potential for proliferation. The United States must address these challenges in order to meet our goals for energy, environmental and economic security. In response, the U.S. Department of Energy, Office of Nuclear Energy (DOE-NE) established the Fuel Cycle Technologies (FCT) program (http://energy.gov/ne/fuel-cycle-technologies).

Long-term resolution of these challenges entails development of sustainable systems that reduce waste while improving resource utilization and safety. To identify potential solutions, the FCT program adopted a results-oriented, science-based approach towards research and development (R\&D) that takes advantage of advances in high-performance computing to integrate theory and experimental data with modeling and simulation.

To achieve its goals and objectives and to provide policymakers the tools to make informed decisions, the FCT program has established five R\&D campaigns and one planning project:

\section{$R \& D$ Campaigns}

- Fuel Cycle Options is developing systematic, transparent, and objective processes to screen and evaluate a wide variety of proposed fuel cycles to identify potential solutions.

- Advanced Fuels is supporting both existing and next-generation reactors by developing accident-tolerant light water reactor fuel and advanced proliferation-resistant fuels for sustainable fuel cycles.

- Material Recovery and Waste Form Development is developing innovative processes to recover uranium and other materials from UNF while improving proliferation resistance, reducing losses, and minimizing waste. This campaign is also seeking transformational breakthroughs in waste forms suitable for geologic repository environments. 
- Used Fuel Disposition is building the scientific foundation and technology for long-term interim storage, transportation, and permanent disposal of UNF and HLW.

- Material Protection, Control, and Accountability Technologies supports security and safeguards by developing tools and techniques to prevent the misuse of nuclear material.

Planning Project:

- The Nuclear Fuel Storage and Transportation Planning Project lays the groundwork for implementing interim storage, including associated transportation, per the 2013 DOE Strategy, and develops a foundation for a new nuclear waste management organization.

The FCT program mission is multi-faceted, tackling current issues with the nation's UNF inventory and maximizing performance and safety of the existing nuclear fleet, while also developing advanced systems for the future that will ensure nuclear energy's continued role as a clean and sustainable energy source. FCT programs goals that are directly addressed by the UFD campaign are in boldface type.

\section{FCT Near-Term Goals}

Strengthen the technical and scientific basis for extended storage of UNF and HLW, and work with industry to develop and demonstrate solutions.

- Identify and test options to enhance accident tolerance of the current reactor fleet.

- Identify and select preferred fuel cycle options that address key challenges, including deployment of advanced uranium enrichment technologies to enhance national energy security.

\section{FCT Medium-Term Goals}

○ Deploy the selected extended storage solution while developing the scientific basis for permanent disposal options in a geologic repository.

- Demonstrate and deploy the selected enhancements for accident tolerance.

- Conduct science-based, engineering-driven research to fully evaluate and characterize the selected sustainable fuel cycle options. ${ }^{1}$

\section{FCT Long-Term Goals}

- Implement safe strategies for management of UNF and HLW, including both storage and permanent disposal solutions.

- Deploy advanced nuclear systems for affordable, safe, and secure nuclear-generated electricity while continuing to test enabling technologies for future deployment.

\footnotetext{
${ }^{i}$ A sustainable fuel cycle is one that: improves uranium utilization, maximizes energy generation, minimizes waste generation, improves safety, and limits proliferation risk.
} 


\section{DOE Strategy for the Management and Disposal of UNF and HLW}

The January 2013 DOE Strategy for the Management and Disposal of Used Nuclear Fuel and HighLevel Radioactive Waste (http://energy.gov/downloads/strategy-management-and-disposal-usednuclear-fuel-and-high-level-radioactive-waste, henceforth referred to as the "DOE Strategy") identifies key goals and target dates associated with used nuclear fuel management. These goals provide a framework for setting priorities within the Used Fuel Disposition Campaign. The budget allocated to the program then determines whether or not the target timeline can be met.

The goals summarized below are identified in the DOE Strategy document, with the caveat that full execution of the plan requires enactment of revised legislative authority.

2021 Pilot interim storage facility opened and operating

- Focus on orphaned fuel at shut-down plants

2025 Consolidated interim storage facility (CISF) opened and operating

- Repackaging capability to provide flexibility in operation of the transportation system and disposal facilities

- Sufficient capacity to allow taking possession of sufficient quantities of UNF to make progress on the reduction of long-term financial liabilities

2026 Permanent repository sited

2042 Repository site characterized; repository designed and licensed

2048 Repository constructed and operations commenced

The DOE Strategy additionally notes “...the Administration understands the need for the Environmental Protection Agency to develop a set of generic, non-site-specific, repository safety standards to gain public confidence that any future repository will protect public health and the environment. This will be an important early step in any repository siting effort."

The DOE High Burnup (HBU) Dry Storage Cask Research and Development Project led by Electric Power Research Institute (EPRI) is a full-scale industry demonstration that will result in data on HBU fuel assembly performance in dry storage conditions. This project also establishes target dates that influence UFD programmatic activities. Key dates for the HBU demonstration are summarized below.

2017 Load fuel into casks at industry site; acquire two gas samples over a two-week period

2018 EPRI contract for HBU demonstration is complete; loaded cask is sitting on a pad with minimal on-going monitoring

2027 Dry storage cask is opened at a DOE site for inspection (must be included in UFD plan)

The UFD Campaign recognizes that the dates established in the DOE Strategy only provide a rough guideline and require legislative action to be enacted. Despite the associated uncertainty in the progression of these steps toward the eventual operation of a repository, this strategy provides a tangible set of goals that can be used in establishing campaign priorities and funding distributions to achieve the steps that will necessarily precede the end-goals summarized above. This document focuses on the UFD mission to conduct research and development activities that provide a technical basis for storage, transportation and disposal activities. The responsibility for implementing storage and transportation decisions lies with the Nuclear Fuel Storage and Transportation Planning Program. 


\section{UFD Campaign Mission and Objectives}

The safe management and disposition of used nuclear fuel and/or high-level nuclear waste is a fundamental aspect of the nuclear fuel cycle. The United States currently utilizes a once-through fuel cycle where used nuclear fuel is stored on-site in either wet pools or in dry storage systems with ultimate disposal in a deep mined geologic repository envisioned. Multiple disposal strategies and long interim storage at reactor sites are being considered. In addition, the Fuel Cycle Technologies Program is investigating alternatives to the once-through fuel cycle and there is a need for a sound technical basis for managing radioactive wastes from any future nuclear fuel cycle to provide acceptable disposition paths for all wastes regardless of origin. These disposition paths will involve both the storage of radioactive material for some period of time and the ultimate disposal of radioactive waste. As disposition paths evolve from the continuing R\&D process, it is important that storage options for fuel cycle materials remain as flexible as possible in order to facilitate selected disposal options.

The storage and transportation of UNF has a stable regulatory basis, is technically mature, and is operationally safe. However, two factors drive the need for R\&D investment:

1) There will be a need for storage licenses to be extended beyond the regulatory time limit.

2) The utility industry has been maximizing plant efficiencies, which has resulted in markedly higher average fuel burnup.

To extend the license time period for onsite storage, data that demonstrate used fuel behavior and characteristics over long periods of time needs to be obtained. For high burnup fuels, data as well as modeling and simulation are needed to demonstrate fuel behavior and characteristics during extended storage, as well as during transportation, with a particular focus on the effects of high burnup on fuel and cladding. While the near term focus is on existing used nuclear fuel, wastes from alternative fuel cycle technologies will also be addressed with appropriate prioritization.

The disposal of radioactive waste of all classifications (low-, intermediate-, high-level waste, and used nuclear fuel) has been investigated worldwide since the inception of nuclear power. While significant progress has been made regarding disposal, the routine disposal of used nuclear fuel and radioactive waste remains problematic. Experience both in the US and internationally has illustrated the challenges of siting, characterizing, designing, and licensing a geologic repository. Progress has been demonstrated by the deployment of near-surface disposal facilities for low-level waste and the Waste Isolation Pilot Plant for the disposal of defense-related transuranic wastes. However, the capacity for disposing of low-level wastes is limited. Details on the characterization of low-level waste as Class A, B or C are provided in 10 CFR Part 61.55. Potential disposal pathways for Greater Than Class C low-level waste (which is essentially intermediate-level waste) have yet to be identified, and the disposal of UNF and HLW has not been demonstrated. Ensuring nuclear energy's continued role as a clean and sustainable energy source requires the availability of routine disposal pathways.

\section{Campaign Challenge}

The challenge to the UFD Campaign is to provide a sound technical basis for supporting the current DOE strategy for managing the back end of the nuclear fuel cycle, including the identification and evaluation of safe and secure options for storage, transportation, and permanent disposal of 
radioactive wastes resulting from existing and future fuel cycles. A sound technical basis for multiple viable disposal options will increase confidence in the robustness of generic disposal concepts and will develop the science and engineering tools needed to support disposal concept implementation. A "sound technical basis" for a specific option would indicate that sufficient information is available for DOE to conclude that licensing can be supported. Additionally, science-based information is needed to support extended storage and eventual large-scale transport of UNF and high-level radioactive waste for future disposal.

The vision of the UFD Campaign includes implementation of strategies and technologies for the safe long-term management and eventual disposal of U.S. commercial UNF and associated nuclear wastes by mid-century. Accomplishment of this vision requires that significant testing, modeling and demonstration activities be completed to establish the necessary technical basis for safe storage, transportation and disposal of UNF, particularly as UNF discharge burnup is increased and as storage times extend beyond what was originally intended.

\section{Campaign Structure}

The Campaign is divided into two primary technical areas intended to aid the accomplishment of the established objectives:

- Storage and Transportation Research

- Disposal Research

Figure 1 provides a work breakdown structure for the UFD campaign, highlighting the two technical areas and the focus areas under each. Specific activities are coordinated with international organizations, other FCT campaigns and the Nuclear Fuels Storage and Transportation Planning Project.

\section{Campaign Mission}

The mission of the UFD campaign is to identify alternatives and to conduct scientific research and technology development to enable storage, transportation and disposal of used nuclear fuel and wastes generated by existing and future nuclear fuel cycles.

Recognizing that the current system for managing UNF and HLW is viable for several decades, the Used Fuel Disposition Campaign has established near-term (5-yr) and medium-term (10-yr) objectives to meet the overarching goals for used fuel and high-level waste management, building on research completed in these areas since 2009. Additional information regarding specific goals associated with these objectives is provided in Section VI.

\section{Near-Term (5-yr) UFD Objectives}

1. Provide technical expertise to inform policy regarding management of UNF and radioactive waste that would be generated under existing and potential future nuclear fuel cycles.

- This will involve continuing to refine our understanding of our current and future inventory and how that inventory can be managed most effectively.

2. Develop the technical basis for extended long-term storage of UNF and high-level nuclear waste to identify long-term research and development needs. 
- Fuel will be stored for longer than originally anticipated; hence, a better understanding of the condition of the fuel, cladding, and containment must be established over the duration of the storage period.

3. Develop the technical basis for retrievability and transportation of high burnup UNF.

- Industry is realizing increased efficiencies by burning nuclear fuels in commercial reactors longer; as a result, the waste inventory will eventually be dominated by high burnup fuel. Potential technical problems that may arise when trying to retrieve and transport older, higher burnup fuel must be investigated.

4. Develop a comprehensive understanding of the current technical basis for disposal of UNF and high-level nuclear waste in a range of potential disposal environments to identify long-term R\&D needs.

5. Develop advanced, predictive computational models, with experimental validation, for:

a. Evaluation of UNF performance under normal conditions of storage and transportation.

b. Evaluation of disposal system performance in a variety of generic disposal system concepts and environments.

\section{Medium-Term (10-yr) UFD Objectives}

1. Support the implementation of a full-scale, NRC-licensed confirmatory storage demonstration facility via significant collaboration with industry.

2. Develop the technical basis necessary to support eventual transportation of UNF.

3. Implement integrated storage, transportation and disposal concepts. 


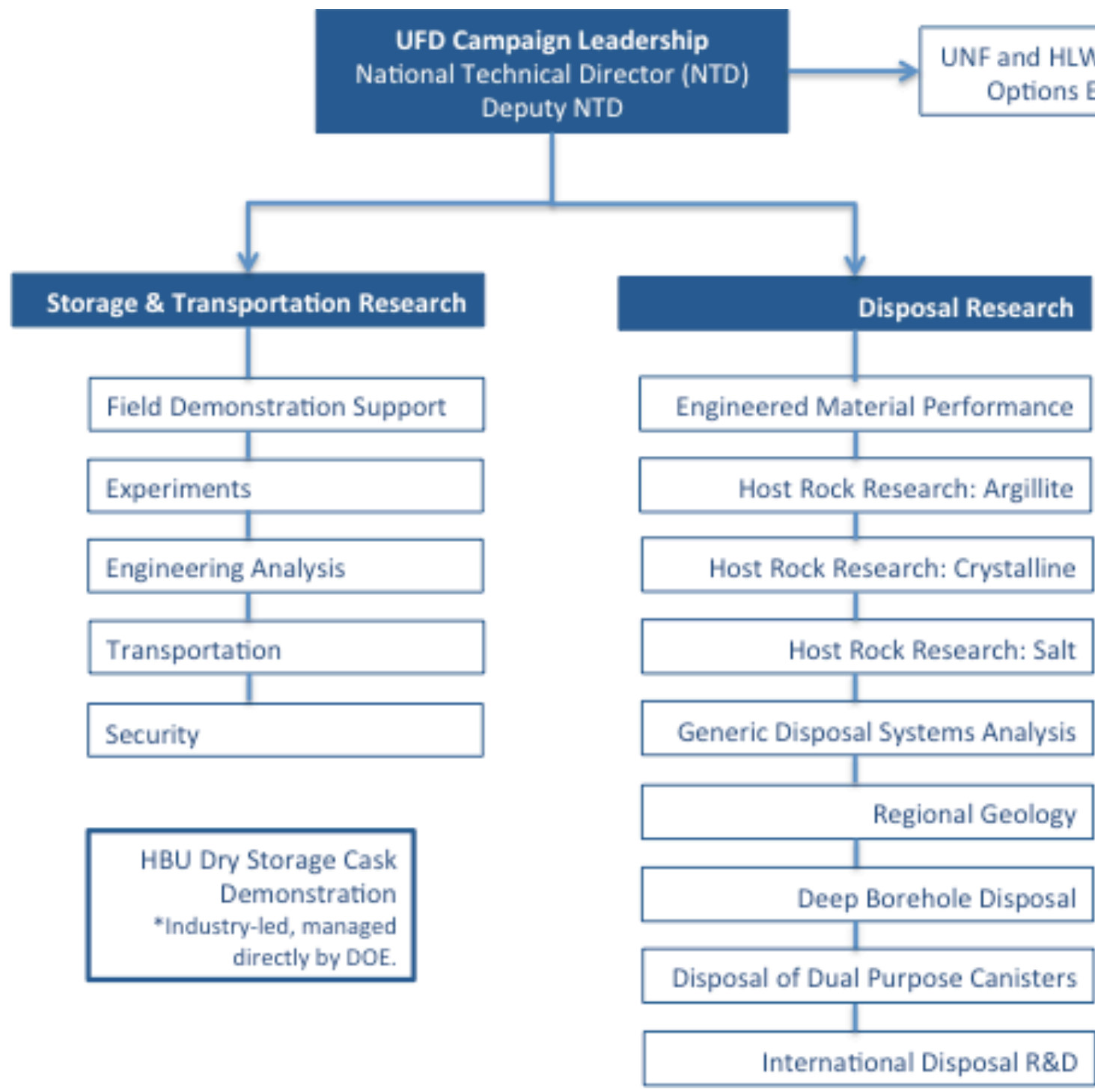

Figure 1. Used Fuel Disposition Campaign work breakdown structure. 


\section{Technical Challenges and Definition of R\&D Objectives}

A detailed description of each of the primary technical research areas within Used Fuel Disposition, Storage \& Transportation (S\&T) and Disposal Research (DR), is provided below. This section identifies key challenges within these technical areas and specifies more detailed goals and objectives that align with the broader campaign goals and objectives defined in section IV.

\section{V.A Storage \& Transportation}

The storage and transportation of UNF has a stable regulatory basis, is technically mature, and is operationally safe. However, extended storage times and increased burnup of commercial fuel at discharge have precipitated the need for additional technical data regarding fuel and storage system performance. Storage and Transportation R\&D focuses on closing technical gaps related to extended storage of UNF. Data on used fuel behavior and characteristics will support the demonstration of safe storage over long periods of time, as well as safe transportation subsequent to storage. For high burnup fuels, experimental data, modeling and simulation will help demonstrate fuel behavior and characteristics during extended storage and transportation.

\section{Storage}

Used LWR fuel is currently being safely and securely stored in the U.S., primarily at the locations where it was generated. It is initially stored in wet used fuel pools, but as these pools fill to capacity the used fuel is transferred to dry canisters that are stored on-site at the nuclear power plant. UNF is also transferred from wet pools to dry storage systems when a nuclear power plant is decommissioned, but, at present, the used fuel remains at the reactor site. High-level radioactive waste, including both liquid wastes from reprocessing of UNF and solid waste forms such as vitrified borosilicate glass generated from liquid wastes, is currently stored in the U.S. at facilities managed by the DOE Office of Environmental Management.

Neither wet nor dry storage systems offer a permanent solution for managing UNF or HLW. Rather, they are designed to be temporary, with plans for ultimate removal of the material and subsequent disposition (i.e., disposal). The unavailability of disposition options, such as a permanent disposal facility, has resulted in the potential need for an extended storage period. Dry used fuel storage systems are typically licensed by the US Nuclear Regulatory Commission (NRC) for a 40-year period, with the possibility of a 40-year license renewal allowing for up to 80 years of operations under current regulations. Over the next decade, these licenses will begin to expire. The UFD Campaign supports the development of the technical basis to judge the viability of extending the storage licenses to allow continued on-site storage.

Current practice provides for safe and secure storage of UNF at existing facilities. This is a reasonable approach based on current economics, a stable regulatory environment, and sufficient land space to expand the on-site storage footprint as needed. Several additional considerations are relevant to the design of a storage $\mathrm{R} \& \mathrm{D}$ program from a longer-term strategic perspective:

- Consolidated Storage. As UNF continues to be stockpiled in long-term storage, on-site storage space will become limited and, consistent with the DOE Strategy, there may be benefits from consolidated storage of UNF at one or more locations. 
- High Burnup UNF Storage. The higher burnup UNF that is typical of current industry practice may require additional assessments to confirm its performance during extended storage.

- Post-Storage UNF Integrity. Validation requirements for UNF integrity for transportation after long-term storage may need to be developed.

- UNF Material Property Data. Experiments to obtain used fuel cladding material property data are inherently expensive and complex, requiring careful prioritization and planning of experimental measurements.

The 5- and 10-year UFD Goals for storage are structured to address these issues so that all fuel cycle material will continue to be stored in a safe and secure manner and in a way that will be adaptable to selected disposal paths.

\section{Transportation}

Significant R\&D and operational experience exists for the transportation of LWR used fuels. However, higher burnup fuels and advanced fuels that may be developed as part of future fuel cycle options will likely require development of an associated material properties database and may require new technologies to support their transportation. Several R\&D topics are associated with safe and secure transportation activities:

- Material Property Understanding. The safety of extended storage and subsequent transportation of UNF must be verified through experimental data collection, associated modeling and simulation, and confirmatory demonstration. In particular, the focus will be on material property data of high burnup UNF cladding.

- Cladding Integrity Investigations. Data regarding the performance of high burnup fuels during transportation are currently limited. Experimental investigations are necessary to characterize the impact of burnup on the strength and ductility of fuel cladding and its performance during transport.

- Over-the-Road Testing. Current gaps in the understanding of forces that UNF experiences during transport motivate the conduct of over-the-road tests on instrumented dummy assemblies to ascertain actual loads that the fuel experiences during transport. This loading information, coupled with the cladding integrity investigations, will allow quantification of fuel response during transport.

- Secure Transportation. As planning continues for larger scale transportation campaigns, the security framework surrounding these campaigns will be evaluated in light of current rulemaking that is advancing through the NRC. The impact of new regulations may require changes in how used fuel is protected during transportation.

\section{Key Storage and Transportation (S\&T) Goals:}

A. Support development of a technical basis to ensure that waste can be moved to and disposed of at the ultimate site of choice. Issues that need to be investigated include:

1) Transport of high burnup UNF;

2) Extended storage of UNF;

3) Retrievability and subsequent transport of UNF after extended storage; 
4) Storage canister integrity.

B. Support the industry-led Full-Scale Confirmatory Demonstration that will generate data during the drying and storage times.

\section{Near-Term S\&T Objectives (5-yr):}

1. Develop a sufficient understanding of how hydrides affect cladding integrity in HBU UNF.

a. Develop a predictive model of hydriding in nuclear fuel cladding that can address degradation phenomena not readily obtainable through experimentation. This model will be validated with experimental data where possible.

b. Conduct experimentation and analysis to develop an increased understanding of pellet-to-pellet and pellet-to-clad interactions and the impact of the related stresses on hydride formation.

c. Perform experiments to develop a better understanding of the effects of temperature and pressure on the Ductile-to-Brittle Transition Temperature.

2. Characterize and develop an understanding of how corrosion and stress corrosion cracking affect the performance of stainless steel storage canisters.

a. Understand the performance of the canister parent material and the closure weld material.

b. Better characterize the on-site environmental conditions in both marine and inland locations.

c. Understand residual stress and related stress corrosion cracking in typical dry storage canisters.

Note: Existing models are expected to be adequate, but confirmatory data is needed for validation.

3. Characterize and understand how external loadings are transferred to UNF during normal conditions of transport. Develop a predictive model validated with experimental data.

a. Work will be performed to determine if the stress induced by normal conditions of transport is greater or less than the maximum stress the material can withstand. This research includes instrumenting surrogate assemblies and subjecting them to the stresses and strains of normal conditions of transport, using both shaker tables and real drives over roads and/or rails.

4. Thermal Profile Analyses

a. Complete analysis of assembly and cask thermal conditions during storage. Data will be collected during on-site environmental monitoring events and during the full-scale demonstration. These models will be used to predict hydride formation, corrosion rates, and other factors that will affect the integrity of the fuel and its packaging.

5. Support the Full-Scale HBU Industry Demo (DOE/EPRI). 
a. Collaborate with industry to identify sister rods, candidate fuel and support basket configuration analysis by 2017 .

b. Support identification of instrumentation for long-term monitoring by 2017 .

c. Identify separate effects test that align:

i. Testing of sister rods to obtain time zero $\left(\mathrm{t}_{0}\right)$ data.

ii. Characterization of fuel rods extracted from the dry storage canister (2027).

d. Identify and develop a plan to initiate necessary facility upgrades by 2027 to handle the dry storage cask and to extract assemblies / fuel rods. It is anticipated that modification of an existing facility would require $\sim 5$ years:

i. 1 year for contracting

ii. 1 year for design

iii. 3 years for construction

Note: Activity would need to start by 2022 to be operational in time to support 2027 evaluation of fuel from the dry cask demonstration.

\section{Medium-Term S\&T Objectives (10-yr):}

1. Develop an improved understanding of the effects of the vacuum drying process for used fuel. Fuel and cladding is exposed to its peak post-irradiation temperature during this process, causing it to be a critical time for many of the factors that affect fuel integrity. Aspects of interest include:

a. Remaining moisture levels;

b. Effect of drying on neutron poisons in the basket;

c. Impact on hydride formation in the cladding;

d. Effect on radiolysis (hydrogen production).

2. Develop a dry transfer system that allows transfer from the current storage environment to interim storage.

a. There are currently over 500 casks at Independent Spent Fuel Storage Installations (ISFSIs) that do not have a licensed transportation cask. Many of these sites do not have pools for cask handling, so a dry transfer system will be needed to transfer the contents to a transportation cask. A dry transfer system will also be necessary if an assembly and/or canister needs to be repackaged due to retrievability concerns.

3. Determine the impact of multiple drying cycles on fuel cladding hydride behavior.

a. If fuel is repackaged due to transportation and storage needs or retrievability, some of that repackaging may occur in pools. This would result in multiple drying cycles. It is important to understand the effects of multiple drying cycles on irradiated and high burnup fuel because the fuel, cladding, and assembly reach the hottest temperatures during drying. Current data suggests that hydride 
formation and fuel and cladding embrittlement are most affected by high temperatures and the subsequent cooling rate.

4. Develop an aging management plan for the long-term inspection of canisters during extended storage.

a. Both industry and the regulator have expressed a need to be able to predict when conditions are suitable for degradation of critical safety components. To do this, research must focus on methods to detect environmental conditions that are conducive to degradation as well as ways to detect the first signs of and then trending of degradation.

b. R\&D Components include:

i. In situ monitoring instrumentation for storage and

ii. Periodic inspection.

5. Support implementation of facility upgrades identified in the short-term objectives to conduct separate effects testing in support of the confirmatory full-scale demonstration of the TN-32 dry storage cask.

Note: Implementation of facility upgrades would likely be conducted outside DOENE53 and the UFD Campaign.

The identified objectives require a number of experimental and modeling activities to be achieved. The current S\&T activities are summarized in section VII.B.

\section{V.B Disposal}

Safe disposal of radioactive waste is accomplished by isolating the waste from the human environment for sufficiently long time periods, as required by regulatory standards. Isolation is achieved through a combination of engineered and natural barriers (e.g., robust waste packages and very slow radionuclide transport through the surrounding rocks), essentially resulting in no radiological risk to the biosphere for many thousands of years when these barriers perform as expected. Over the very long time periods necessary to isolate the wastes, the engineered barriers may degrade, allowing radionuclides still present in the waste to be released at a low rate into the natural system. The natural system will act to reduce and delay the subsequent migration of radionuclides to the accessible environment to levels established in regulatory standards, typically well below naturally occurring levels of background radiation. Understanding when radionuclides may begin to be released from the disposal system, the rate at which they may be released, and how they might migrate through the natural system are primary factors in demonstrating the safety of a disposal system concept.

Geologic disposal concepts are typically complex systems with physical and chemical couplings between the disposed waste, engineered barrier materials, and the natural system. The long-term radionuclide isolation capabilities and characteristics of a waste disposal environment are dependent on the details of the site and the form and contents of the wastes to be disposed. Disposal systems can be geographically large and the distance over which materials could potentially migrate can be long. In addition, because of the long time frames involved, precise predictions of repository performance are not possible, and uncertainty must be acknowledged in the decision-making process. Quantifying, propagating, and reducing this 
uncertainty through safety assessments have been and continue to be challenges in demonstrating the viability and safety of geologic disposal. Disposal R\&D focuses on gathering sufficient data (laboratory and field) and developing computational models for evaluating and demonstrating long-term disposal system performance and safety.

Evaluations indicate that, based on existing information, reasonable designs, and generic safety assessment models, it is likely that mined geologic repositories (i.e. in salt, clay/shale rocks, or crystalline rocks) or deep borehole disposal facilities that are capable of safely isolating used nuclear fuel and high-level radioactive waste from the public and the environment can be developed in the U.S.

The overarching goals and objectives for disposal research are summarized below. These more detailed objectives align with the broader program objectives identified in section IV.

\section{Key Disposal Research (DR) Goals:}

A. Provide a sound technical basis for multiple disposal options in the U.S. for existing and reasonably projected future UNF from the existing commercial LWRs and the DOE managed UNF and HLW (before 2026).

1) A "disposal option" is defined to include waste forms, waste form packaging, disposal system design (including both mined repositories and deep boreholes) and host medium. The disposal system is defined to include both the engineered and natural systems.

2) International research provides confidence that proposed mined repository options are viable for the major host rocks (e.g., crystalline rock, salt, clay/shale). Field testing remains necessary to confirm the feasibility of deep borehole disposal.

3) Site Selection is a decision that is made based on scientific, engineering, and sociopolitical factors; technical work is necessary to inform site selection.

4) Viability of a specific concept is dependent on the design details suited for the specific host medium.

B. Capture and quantify the advantages and disadvantages of each generic disposal option using representative reference cases.

1) This work is necessary to increase confidence in the robustness of generic disposal concepts.

2) The natural barrier system, engineered barrier system (including waste package and waste forms), and repository layout must be included in the evaluation.

3) Thermal load management is a key consideration in disposal system design.

4) Major epistemic and aleatory uncertainties should be captured and quantified.

C. Develop science and engineering tools to support disposal concept implementation (by 2026, per the DOE Strategy).

D. Provide the necessary technical information for a successful site-specific license application. Note: Achieving this goal requires decisions to be made outside the scope 
of the UFD Campaign. Supporting work must be performed after site selection in 2026, per the DOE Strategy.

\section{Near-Term DR Objectives (5-yr):}

1. Update the R\&D Roadmap and the Generic Deep Geologic Disposal Safety Case for UFD DR:

a. Organize current information, including international data.

b. Considering work accomplished to date, determine information gaps for each disposal media.

c. Develop a prioritized gap analysis for each disposal medium.

2. Complete evaluation of the direct disposal of dual-purpose canisters (DPCs).

3. Complete a Geographical Information System (GIS) database to support site screening and selection.

4. Develop an experimental and modeling basis for understanding the long-term performance of engineered materials, including waste form and waste packaging, clay buffers, and backfills at elevated temperatures.

5. Initiate a full-scale deep borehole field test that will provide basic data to evaluate feasibility of deep borehole disposal of HLW and DOE-managed UNF.

6. Develop an experimental and modeling basis for understanding the long-term performance of:

a. Argillaceous host rock (clay-rich)

b. Crystalline host rock

c. Salt host rock, Domal or Bedded

d. Deep boreholes

7. Develop system analysis tools to support generic technical site characterization, site screening, and prioritization of data needs.

8. Develop a process to capture and quantify realistic advantages and disadvantages of each disposal option. Reach consensus on realistic reference cases for each disposal option.

9. Develop a process for generic technical characterization of volunteer and/or selected sites. Develop a functional criteria document.

10. Leverage, and potentially partner with, current and future work conducted by international partners.

\section{Medium-Term DR Objectives (10-yr):}

1. Provide technical input to advantages and disadvantages of specific volunteer sites. (Required timeframe: Must be complete by 2026 to meet the DOE Strategy.)

2. Design and implement a site characterization program for one or more sites. (Required timeframe: 2026 - mid-2030s) 
3. Complete a full-scale deep borehole field test, including demonstration of waste canister emplacement using surrogate waste forms.

4. Establish generic or site-specific underground research laboratories (URLs) to conduct research and demonstration tests.

5. Develop site-specific licensing basis for one or more sites. (Required timeframe: Complete by 2042)

6. Design and implement a specific performance confirmation program for each of one or more sites. This is an experimental program necessary to confirm the licensing basis, which must continue throughout operation of the repository. (Required timeframe: 2042 $-2048)$

The identified objectives require a number of experimental and modeling activities to be achieved. The current Disposal Research activities are summarized in section VII.C. 


\section{Research Necessary to Meet DOE Strategy Goals}

In order to meet the goals set out in the DOE Strategy for the management and disposal of UNF and HLW it is necessary to recognize that specific research and development tasks must be completed within a defined timeline. This section identifies an approximate timeline and tasks required to meet the stated goals. Note that dates established in the EPRI HBU dry storage cask demonstration project are also incorporated into the summary of R\&D needs. Some items listed below are not specifically research and development tasks but must be completed in order to achieve the stated goal. Many of the non-R\&D tasks fall to the responsibility of the Nuclear Fuel Storage and Transportation (NFST) Planning Project. Although these tasks are outside the scope of UFD, it is necessary to understand how UFD and NFST are interrelated. Simultaneously tracking tasks under UFD and NFST will ensure that the programs maintain focus on high priority items to meet the long-term DOE Strategy goals. Specific R\&D items are marked with a diamond bullet notation:

* R\&D task.

2017 Load fuel into casks at industry site; acquire two gas samples over a two-week period (HBU storage demo)

Requirements:

- Laboratories must collaborate with industry to identify candidate fuel for inclusion in the demonstration, including selection of sister rods for analysis prior to the storage period.

- Laboratory personnel perform analysis of the planned cask support basket configuration.

- Laboratory personnel support identification of instrumentation for long term monitoring.

2018 EPRI contract for HBU demonstration is complete; loaded cask is sitting on a pad with minimal on-going monitoring (HBU storage demo)

2021 Pilot interim storage facility (CISF) open and operating

- Focus on orphaned fuel at shut-down plants

Requirements:

- Licensing basis established for fuel retrieval from dry storage casks (low BU) - [2-4 yr process, start 2017]

- Design and build licensed rail car (operating assumption is arrival by rail in a rail cask with some heavy-haul transport to nearest rail from some sites)

- Pilot site selected using consent-based siting

- $\quad$ EIS / FEIS completed [minimum 2 yr process, start 2018]

- Site characterization complete

- Design, license, build and plans for operation complete

- CISF licensed for operation [2-4 yr process (2, DOE; 3-4, NRC), start 2017]

* Validated model results submitted to license representative [by 2018]

- Provide expected performance analysis results to license representative to inform license application [by 2017]

* Predictive modeling tools validated

- Tools available and validated with measured experimental data to the extent possible

- Quality Assurance (QA) program established 
2025 Consolidated interim storage facility (CISF)

- Repackaging capability to provide flexibility in operation of the transportation system and disposal facilities

- Sufficient capacity to allow taking possession of sufficient quantities of UNF to make progress on the reduction of long-term financial liabilities

Requirements:

- Completed design for a handling and packaging facility consistent with disposal plan

* Licensing basis developed for the storage and transportation of HBU fuel in standard DPCs [by 2021]

* Licensing basis developed for fuel retrieval from dry storage casks (HBU) [by 2021]

- $\quad$ EIS / FEIS completed [minimum 2 yr process, start 2022 ]

- Design, license, build and operating plans complete

- Provide expected performance analysis results to license representative to inform license application [by 2018]

* Validated models available [by 2019]

- Predictive modeling tools available and validated, to the extent possible, with measured experimental data for HBU fuel

- QA program established

\section{Permanent repository sited}

Requirements:

* Completed GIS database [by 2018]

* Defined site characterization process [by 2018]

- Site screening and characterization process defined and implemented

- Features, events and processes (FEPs) defined for each site

- Defined inventory of what will go into the repository

- Include detailed attributes of waste forms as intended for disposal

- Defined list of candidate sites [2022]

- Defined down-selection process from volunteer sites [2026]

* Predictive modeling tools validated [by 2022]

- Data collected for specific candidate sites

- Preliminary conceptual repository design to perform analysis

* Metrics for a suitable site evaluation defined [by 2020]

- Performance measures that will be calculated by the model

- Metrics may be a reiteration of NRC-defined regulations for a repository

- Considerations: dose, thermal load, cost, time, etc.

- Cost analyses

- QA program in place

- EIS started

2027 Dry storage cask from industry demonstration is opened at a DOE site for inspection (HBU storage demo)

Requirements:

- Required facility upgrades for cask handling, fuel removal and fuel characterization complete. 
- Separate effects test plan prepared. Test plan must align characterization of sister rods (time zero, $t_{0}$, property determination) with characterization of the fuel rods post-storage.

$2038 \quad$ Repository site license application submitted

$2042 \quad$ Repository designed and licensed

Requirements:

* Site characterization complete [by 2034]

- Iterative process with system performance analysis

**Typically plan for $\sim 15$ yrs, but this does not fit within the current estimated timeline.

- Underground Research Laboratory testing

* Understanding of data and processes is sufficient to support licenses

[complete by 2034 to support license application; work will continue through licensing approval]

- Repository design and plans for operation sufficient for licensing

* Iterative process with system analysis [2026 - 2034]

- Freeze design in 2034

* Pre- and post-closure safety analyses

- Model must be available prior to this step [model evolves 2026-2034]

- Post-closure analysis as a component of preparing the license application [20342038]

* Performance confirmation program in place [defined by 2038; implemented through closure and post-closure monitoring]

○ Includes instrumentation and monitoring plan in place

- Assess what instrumentation is available at present and when would need to be available

- License in-hand

○ Milestones for licensing:

- Submit license [4-yrs to license; submit by 2038]

- Complete characterization [4-yrs to prepare license application; complete characterization by 2034]

- Site characterization [2026 - 2034, defined by established timeline rather than by duration desired by technical staff]

- $\quad$ Detailed design in-hand

- Surface facilities designed

- $\quad$ FEIS completed

\section{Repository constructed and operations commenced}

Requirements:

- Construction and startup schedule must be maintained

- Note: Yucca Mountain assumed that the first waste shipment would be received 9 yrs after license was in hand. 


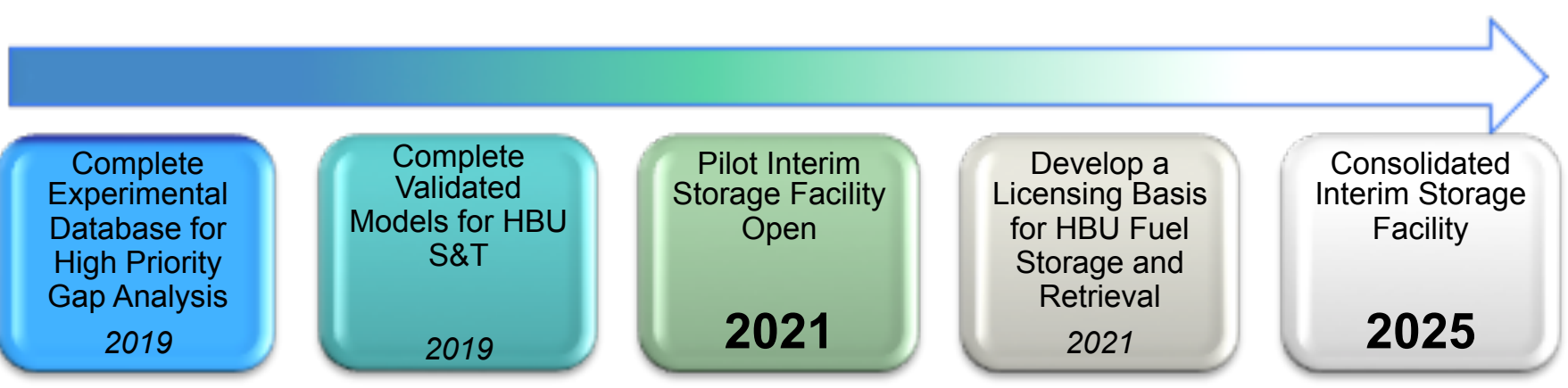

Figure 2. Summary timeline for Storage and Transportation Research activities.

Note: Approximate target dates (in italics) needed to meet deadlines (in bold) set out in the 2013 DOE Strategy.

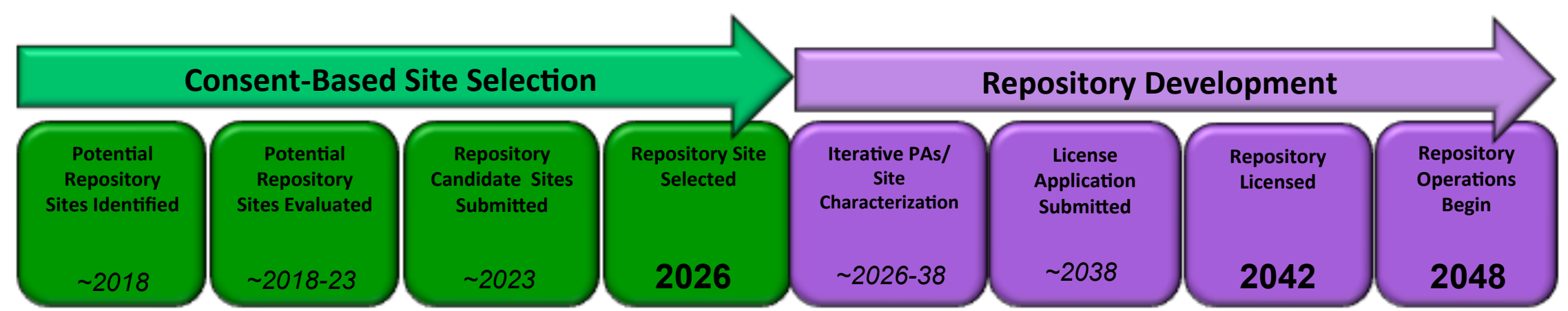

Figure 3. Summary timeline for Disposal Research activities.

Note: Approximate target dates (in italics) needed to meet deadlines (in bold) set out in the 2013 DOE Strategy. 


\section{Current Campaign Activities}

Campaign activities are organized based on the work breakdown structure illustrated in Figure 1. In addition to the overarching campaign management and integration activities performed under the direction of the campaign National Technical Director (NTD) and Deputy NTD, campaign research and development is organized into two technical areas:

1. Storage and Transportation (ST): Activities specifically associated with the storage and transportation of radioactive wastes.

2. Disposal Research (DR): Activities specifically associated with the disposal of radioactive wastes.

A summary of the current campaign activities follows.

\section{VII.A Campaign Management and Integration}

This activity supports campaign management and integration activities, including cross-cutting R\&D. Specific responsibilities include:

- Ensure technical R\&D work throughout the campaign meets quality assurance requirements established by the Fuel Cycle Technologies program and meets programmatic requirements;

- Integrate R\&D activities across the campaign and with other campaigns and industry participants in the Fuel Cycle Technologies program;

- Interact with the Nuclear Energy Advisory Committee, the Nuclear Waste Technical Review Board, and other external advisory and review groups as needed; and

- $\quad$ Support the DOE-NE University Programs as needed.

High-level activities of significant importance to the campaign are managed directly under the management and integration area.

\section{UNF and Disposal Options Evaluation}

This work scope is conducted directly under the campaign management area (as shown in Fig. 1). The overall objective of the work is to evaluate potential impacts of existing UNF and HLW on the feasibility and performance of various disposal options. The study scope includes all existing UNF from commercial, defense, and research reactors, and UNF from reasonably foreseeable operations of existing reactors. The study also includes existing HLW (e.g., vitrified HLW at Savannah River and West Valley) and waste forms projected to be generated in the future from existing process waste (e.g., vitrified HLW from Hanford, Savannah River and Idaho National Laboratory). The study may include consideration of waste forms that are not currently planned for direct disposal without further treatment (e.g., calcine waste at Idaho National Laboratory) or alternatives to planned treatments. Additionally, scope will include investigation into the potential considerations relevant to the commingling of DOE-managed UNF and HLW in a common repository, and specific to the criteria of health and safety, transportation, and national security. 


\section{VII.B Storage \& Transportation}

\section{ST - Field Demonstration}

The objectives of the Field Demonstration control account are to plan and deploy a confirmatory storage demonstration that will validate our understanding of component material degradation issues that are important to safety. This effort will be conducted in close collaboration with industry and the NRC (see section VI.D on the industry-led high burnup dry storage cask demonstration project).

High priority storage component safety issues are identified in the Used Nuclear Fuel Storage and Transportation Data Gap Prioritization report (FCRD-USED-2012-000109). These issues include the collaboration with industry to plan for fielding a demonstration platform(s) under dynamic and constrained conditions. Work will specifically address potential paths to fielding a demonstration platform based on input from industry, the Blue Ribbon Commission recommendations, the DOE Strategy for the management and disposal of UNF and HLW, Congressional guidance, and other input as applicable.

Current activities include:

- Support the industry-led HBU full-scale dry storage demonstration (see section VI.D), specifically in the areas of fuel transfer, instrumentation and analysis.

- Develop the strategy for fuel pin receipt, characterization, sample allocation for the demonstration sister pins.

- Initiate readiness activities to prepare for receipt of sister fuel pins.

- Coordinate dry cask storage instrumentation development.

- Interface with DOE and industry to develop a dry transfer system.

- Coordinate with EPRI for storage canister in-service inspections.

- Conduct concrete degradation and stress corrosion cracking (SCC) detection, imaging and characterization studies.

- Update of the Storage \& Transportation R\&D Plan.

\section{$S T$ - Experiments}

The objectives of the Storage and Transportation Experiments control account are to conduct the separate effects tests (SETs) and small-scale tests (SSTs) that have been identified in the Used Nuclear Fuel Storage and Transportation Data Gap Prioritization report (FCRD-USED-2012000109). As the experimental work evolves, new issues may arise as artifacts of the test programs. This activity will assess the impact on the developing technical basis as new issues arise and will also closely coordinate with relevant regulatory progress to ensure that all $\mathrm{R} \& \mathrm{D}$ activities relate to eventual licensing. This activity will provide complementary data to support the field demonstration activity.

Current activities include:

- Establish process to receive irradiated UNF from utilities at a national laboratory for characterization and sample preparation. 
- Design an appropriate small-scale test SST vessel for testing and evaluation of irradiated fuel in a hot cell.

- Characterize and evaluate cladding properties, e.g.:

- Develop and test non-destructive examination techniques to characterize hydrides in fuel cladding. Irradiation and evaluation of non-hydrided and pre-hydrided cladding.

- Conduct fuel bend tests on unirradiated, irradiated, non-hydrided, and pre-hydrided cladding samples to determine effects of each factor on cladding properties.

- Develop post-irradiation analysis tools and techniques for HBU fuel examination (e.g. fission gas analyzer).

- Support development and implementation of direct disposal of dry storage canisters in a geologic repository.

- Evaluate dry storage canister performance via in-service inspections, examination of weld samples, corrosion testing, stress corrosion cracking, etc.

\section{ST - Engineering Analysis}

The objectives of the Storage and Transportation Engineering Analysis control account are to conduct analyses, integrate experimental data, and develop the technical basis for extended long term storage and subsequent transportation of used fuel. This work includes developing predictive models of materials behavior and determining if the safety-significant components will maintain their ability to meet their safety functions when subjected to various stresses, loads, and vibrations (i.e., stress and thermal profiles). Analyses will serve to augment the experimental work by addressing degradation phenomena not readily obtainable through experimentation, such as hydrided behavior in cladding and low temperature creep over very long periods of time.

Current activities include:

- Address various technical gaps via model development to augment experimental work. Key technical gaps include: thermal profiles, stress profiles, drying issues, hydride reorientation and embrittlement in cladding, cladding creep, and annealing of radiation damage in cladding.

- Support the development of thermal profiles associated with in-situ dry storage systems, including analysis of canister thermal profiles to inform canister corrosion vulnerabilities. Conduct analyses to define fuel thermal characteristics to support assembly selection for the industry HBU dry cask demonstration.

- Support the development of stress profiles for the evaluation of fuel performance subjected to storage and transportation mechanical loading environments.

- Initiate an Uncertainty Quantification (UQ) effort to complement the prioritization reflected in the technical data gap report. The UQ effort will focus on developing a methodology to provide guidance on what experimental data will have the greatest impact on meeting the ST R\&D objectives. 


\section{ST - Transportation}

The objectives of the Transportation control account are to address identified high priority technical issues associated with the retrieval and subsequent transportation of UNF from dry storage facilities. Research includes developing the technical basis for the transport of high burnup used nuclear fuel (greater than $45 \mathrm{GWD} / \mathrm{MTU}$ ) and the transport of all UNF after long-term storage. This work is closely integrated with the ST Experiments and ST Engineering Analysis Control Accounts in obtaining cladding material properties and assessing cladding performance in transit. Work also includes field testing to assess realistic loading on fuel rods and assemblies during normal conditions of transport (NCT) to obtain data needed to evaluate fuel integrity.

Current activities include:

- Analyze of shaker table test data for over-the-road normal conditions of truck transport.

- Conduct shaker table testing for normal conditions of rail transport.

- Collaborate with industry to ship an instrumented surrogate "dummy" PWR assembly to conduct shaker table tests using the vibration/shock response spectra obtained from the transport.

\section{ST-Security}

The objectives of the Security control account are to assess the impact of the self-protection threshold over long storage periods and to assess how material attractiveness issues affect physical protection strategies and requirements. The NRC is currently in rule-making for storage security; hence, UFD storage security work will continue to integrate regulatory changes as they occur. In addition, planning for a systems approach to transportation security will be initiated to understand relative vulnerabilities of various operational phases of transportation.

Current activities include:

- Assess the regulatory rule-making process and changes being considered in the physical protection and security requirements for storage and transportation.

- Assess the regulatory rule-making process and impact of potential changes in light of the need to de-inventory orphaned sites by transporting the used fuel to a consolidated storage facility.

\section{VII.C Disposal Research}

\section{DR - Specific Host Rock R\&D}

The objectives of the Host Rock R\&D activities are to develop experimental and computational capabilities and to perform modeling analyses to support the development of disposal concepts in various geologic media in the near- and far-field environment of disposal. Understanding interactions occurring between fuel waste and various natural and engineered barriers within various design concepts is important for determining design flexibility and optimization, establishing requirements for waste form performance, and providing a scientific basis underlying the safety cases for optional disposal systems. 


\section{Host Rock Research: Argillite (clay-bearing host rock)}

The argillite work package is focused on the evaluation of important processes in the analysis of disposal design concepts and related materials for nuclear fuel disposal in this type of claybearing repository media.

Current activities include:

- Model development, including

○ Develop constitutive relationships for coupled processes.

- Evaluate relevant physico-chemical processes for clay/shale disposal.

- Develop thermodynamic databases and modeling approaches for chemical equilibria and sorption with insights from experimentally-based studies.

- Develop process models to quantify the effects of chemical, electrochemical, and radiolysis reactions; physical mass transport processes; and environmental conditions on fuel matrix degradation, radionuclide release rates, and overpack performance.

- Conduct clay damage and discrete fracture studies.

- Establish a scientifically sound thermal limit on argillite host rock via high temperature laboratory tests.

\section{Host Rock Research: Crystalline}

The crystalline work packages are aimed at advancing our understanding of long-term disposal of used fuel in crystalline rocks and to develop necessary experimental and computational capabilities to evaluate various disposal concepts in such media.

Current activities include:

- Experimentally investigate structure-functionality relationships of radionuclide transport through and interaction with natural and engineered materials, with a focus on developing a new generation of buffer materials for nuclear waste isolation and establishing new thermal limits for the materials.

- Investigate the stability of intrinsic Pu colloids in crystalline disposal environments.

- Develop models for fluid flow and radionuclide transport in fractured crystalline rocks and for thermal-hydrological-mechanical-chemical behaviors.

- Quantify the long-term weathering behavior of the used fuel matrix (as a waste form) in generic crystalline (granitic) disposal environments.

- International collaboration:

- Collaborate on the Korean Atomic Energy Research Institute (KAERI) Underground Research Tunnel (KURT) testing.

- Collaborate through DEvelopment of COupled models and their VALidation against EXperiments (DECOVALEX), an international cooperative research project on mathematical models of coupled thermal-hydrological-mechanical processing for safety analysis of radioactive waste repositories. 


\section{Host Rock Research: Salt}

The focus of this activity is to use laboratory studies to reduce uncertainties and to enhance the technical bases for disposal of heat-generating waste in salt. Improved data will be developed by experiment for thermal-mechanical behavior of granular salt, its thermal conductivity as a function of porosity and temperature, thermal-mechanical testing of intact and mined salt, brine migration studies, and thermodynamic properties of brines, salt, and corrosion products.

Current activities include:

- Perform thermo-mechanical testing.

- Conduct brine migration studies:

- Perform experiments at varying temperature and pressure conditions.

- Develop constitutive models; benchmark models to test data.

- Investigate the potential for direct disposal of electrorefiner salt in a generic salt-based repository, with a focus on transportation criticality, primary container and overpack requirements, and salt / container chemical interactions.

\section{DR - Generic Disposal Systems Analysis}

The objective of the Generic Disposal System Analysis activity is to develop the necessary system model architecture to support the evaluation of post-closure risk. Modeling will be at sufficient rigor to support the intended use and maintain the flexibility in the system model architecture to meet the evolving needs of the DOE NE and UFD missions. Models will have the capability to produce risk information throughout the potential future phases of the mission, including prioritization of R\&D needs, down selection of geologic disposal options, and site selection and screening. The generic disposal system modeling and analysis capability, including uncertainty, will cover a range of disposal options (e.g., salt, argillite, crystalline, deep borehole).

Additionally, the UFD Disposal Research R\&D Roadmap is an evolving document that will ensure that the technical information needed to implement new national policy for managing the back end of the nuclear fuel cycle is available when decisions are made to move forward. It is focused on generic research and development work undertaken today that will support future site-specific work. As DR continues to evolve, it is necessary to update the R\&D Roadmap to provide both tactical and strategic guidance for the direction of DR. The DR R\&D Roadmap will be updated and managed under the Generic Disposal Systems Analysis activity.

Current activities include:

- Develop a source term conceptual model applicable to all generic disposal options.

- Integrate updated conceptual models of subsystem processes and couplings developed under other work packages; leverage existing computational capabilities.

- Develop and simulate reference cases for generic salt, argillite, and crystalline (granitic) disposal systems.

- Review and update of the DR R\&D Roadmap. 


\section{DR - Deep Borehole Disposal}

The objective of the Deep Borehole Disposal activity is to advance the deep borehole disposal technical basis needed to demonstrate the viability of this disposal concept and make progress towards implementing a full-scale demonstration.

Current activities include:

- Utilize the regional geology database to support site selection for the deep borehole field test.

- Evaluate sub-regional geology and other information for a drilling demonstration project.

- Develop reference designs for disposal of alternative waste forms.

- Evaluate borehole seals for chemical, mineralogical and physical stability in the deep borehole environment.

Planned activities include initiation of a field demonstration test to evaluate the feasibility of the deep borehole disposal concept, conditional on programmatic approval. Specific activities associated with the first year of a deep borehole field demonstration test using surrogate waste will include:

- Establishment of a project management structure.

- Preliminary characterization and evaluation of one or more candidate sites for a field test.

- Selection of a site for a field test, including land acquisition.

- Development of a conceptual design and requirements for borehole construction.

- Development of a conceptual design and requirements for disposal canisters and waste handling.

- Evaluation of regulatory and legal requirements, including obtaining necessary permits.

- Modeling to support site evaluation and test design.

\section{DR - International Disposal R\&D}

The International Disposal control account is established to ensure the integration and coordination of UFD-supported activities among participating national laboratories and comparable programs outside the US. UFD management will be advised of international opportunities that complement ongoing campaign $\mathrm{R} \& \mathrm{D}$ and of activities that have the greatest potential for substantive technical advances. International collaboration is a beneficial and cost-effective strategy for advancing disposal science with regards to multiple disposal options and different geologic environments.

\section{VII.D High Burnup Dry Storage Cask Demonstration}

A DOE-led project was also funded in FY 2013 to establish a high burnup dry storage cask demonstration project. Awarded to an industry team led by EPRI, the project will collect data from a UNF dry storage system containing high burnup nuclear fuel. Along with other relevant modeling, separate effects tests, and small-scale tests, the data that will be collected in this demonstration project can be used to confirm the understanding of material degradation over many decades. 


\section{Performance Measures}

Quantifiable performance measures for the Used Fuel Disposition Campaign are defined as milestones associated with the completion of documentation for each of the described objectives. Most of the objectives discussed previously can be appropriately characterized as level 2 or 3 milestones, and will be defined in detail in control account and work package documentation.

\section{Campaign Interfaces}

Within the Fuel Cycle Technologies Program, the Used Fuel Disposition Campaign is intimately linked with the following campaigns:

- Separations and Waste Forms: This campaign is investigating the possible waste forms that could be used to isolate wastes generated under alternative fuel cycles.

- $\quad$ Fuel Cycle Options: UFD provides the Fuel Cycle Options Campaign with information for evaluating waste management impacts within fuel cycle system analyses.

The Used Fuel Disposition Campaign additionally interfaces with:

- The Storage and Transportation Planning Project due to the linkage between storage and transportation implementation in that project and Storage \& Transportation R\&D in the UFD Campaign.

- The DOE Office of Environmental Management because disposal options developed by the DOE Office of Nuclear Energy must accommodate the used nuclear fuel and high-level radioactive wastes, including those derived from defense-related programs that are currently managed by the DOE Office of Environmental Management.

- The Material Protection, Control, and Accountability Technologies (MPACT) campaign to integrate the specific activities related to security of storing and transporting used nuclear fuel with the broader MPACT mission of developing innovative technologies and analysis tools to enable next-generation nuclear material management for future U.S. nuclear fuel cycles;

- The Advanced Fuels Campaign to identify advanced fuel and cladding design options that may be problematic from the perspective of waste disposal early in the development process.

- Industrial partners in both the disposal and storage areas. In the area of storage, UFD currently participates as a member of the Electric Power Research Institute's Extended Storage Collaboration Program and will collaborate with both nuclear utilities and dry storage vendors in the development of field demonstration programs. In the disposal area, UFD collaborates with industry resources having expertise related to geologic disposal to augment the knowledge base within the campaign. In deep borehole disposal, UFD collaborates with an industrial partner having expertise in deep drilling and exploration services. In Salt R\&D, UFD collaborates with an industrial partner having expertise in mechanical testing of geologic media. Additional disposal related industrial collaborations will likely occur. 
- $\quad$ Universities, directly and indirectly, through the Nuclear Energy University Program (NEUP).

The Campaign participates extensively with international working groups. Collaboration with Japan is conducted through the U.S. - Japan Joint Nuclear Energy Action Plan, Waste Management Working Group, and collaboration with the Republic of Korea is through the Joint Fuel Cycle Studies, Fuel Cycle Alternative Working Group bilateral agreements.

The Campaign also supports active cooperation on disposal research in partnership with DECOVALEX (multiple countries); the European Commission Seventh Framework Program; the Nuclear Energy Agency "Salt Club"; the Mont Terri Project (Switzerland); Colloid Formation and Migration Project (Switzerland); the Full-scale Engineering Barrier Experiments (FEBEX) at the Grimsel site (NAGRA, Switzerland); BGR - Federal Institute for Geosciences and Natural Resources (Germany); and the Korean Atomic Energy Research Institute (KAERI) Underground Research Tunnel (KURT, Republic of Korea). DOE's formal membership in these multi-national initiatives provides access to field data and allows participation in ongoing field experiments. 